\title{
HEAT FLUX DURING DIP-COATING OF A SUPERHEATED SUBSTRATE
}

\author{
K. Schweikert, A. Sielaff, E P. Stephan*
}

\author{
Institute for Technical Thermodynamics, Technische Universität Darmstadt, \\ Alarich-Weiss-Str. 10, 64287 Darmstadt, Germany
}

*Address all correspondence to: P. Stephan, Institute for Technical Thermodynamics, Technische Universität Darmstadt, Alarich-Weiss-Str. 10, 64287 Darmstadt, Germany, E-mail: pstephan@ttd.tu-darmstadt.de

Original Manuscript Submitted: 10/7/2019; Final Draft Received: 11/25/2019

\begin{abstract}
We report transient heat flux calculations based on temperature measurements during dip-coating of a superheated substrate. During the withdrawal of the substrate from a pool of volatile liquid, a film of finite length forms on the substrate's surface, locally reducing the substrate temperature due to evaporation. The surface temperature of the solid substrate is measured using high-resolution infrared thermography and used as a boundary condition to calculate the transient heat flux profiles at the interface between the superheated substrate and the fluid. The shapes of these heat flux profiles are analyzed with special focus on the local heat flux in the thin film region and near the three-phase contact line. It is shown how the heat flux in both regions is dependent on wall superheat and dewetting velocity. Two evaporation regimes, namely contact line evaporation and microlayer evaporation, can be clearly distinguished by their magnitude in overall heat flux. A temperature-dependent critical velocity separates both regimes. The local heat flux in the contact line region sharply increases, when the critical velocity is exceeded. Within the thin film, the local heat flux increases with growing wall superheat and decreases with growing dewetting velocity.
\end{abstract}

KEY WORDS: dip-coating, microlayer, contact line, evaporation, heat flux, thin film

\section{INTRODUCTION}

In dip-coating, a sample is withdrawn from a pool of liquid and a thin liquid film is left on the solid surface. Due to the relative motion between fluid and solid surface, this withdrawal process is commonly referred to as a dewetting situation. If the liquid perfectly wets the solid surface, the thickness of this film depends on the liquids' viscosity $\mu$, density $\rho$, surface tension $\sigma$, and dewetting velocity $u$. This dependency was first described by Landau and Levich (1942). If the liquid does not perfectly wet the surface, film formation is only observed once $u$ exceeds a threshold value, which is referred to as the critical dewetting velocity $u_{\text {crit }}$ (Hocking, 2001). For a range of liquids and nonevaporative systems, this process is generally well understood and correlations exist to account for parameters such as wall roughness, coatings, or surfactants (Rio and Boulogne, 2017). Most dip-coating processes, however, aim at drying the film on the substrate and use volatile solvents. If evaporation occurs during the coating process, the film has a finite length $h$ due to liquid mass lost by phase change. Qu et al. (2002) investigated this problem, finding the scalings laws for the length $h \sim u^{5 / 3}$ and the film thickness $\delta \sim u^{2 / 3}$, which are explained by the balance between the input mass flux $M$ and the evaporative mass flux $J$ in the film region. Berteloot et al. (2008) put forward the theory that a minimum coating thickness can be achieved at $u=u_{\text {crit }}$. In Schweikert et al. (2019) the authors varied the temperature of the wall, thereby controlling the evaporation rate. The scaling laws of Qu et al. (2002) could be confirmed. An increase in wall superheat $\Delta T$, the difference between the local wall temperature $T_{w}$ and the saturation temperature $T_{\text {sat }}$, leads to an increase of $u_{\text {crit }}$ and a decrease of $\delta$ and $h$.

The interest in the heat flux distribution at evaporating thin liquid films is driven in particular by boiling research. In nucleate boiling, thin liquid films with a thickness of a few micrometers have been observed underneath growing vapor bubbles (Kim and Buongiorno, 2011; Utaka et al., 2018), feeding the bubble with vapor for its further growth. 


\begin{tabular}{|llll|}
\hline & & & \\
& & & \\
$c_{s}$ & SOMENCLATURE & \\
$h$ & microlayer length & $T_{C L}$ & temperature in the contact line region \\
$J$ & evaporative mass flux & $T_{s a t}$ & saturation temperature \\
$k$ & liquid thermal conductivity & $T_{w}$ & temperature of the wall \\
$k_{s}$ & solid thermal conductivity & $u$ & dewetting velocity \\
$M$ & liquid mass flux & $u_{\text {crit }}$ & critical dewetting velocity \\
$P$ & capillary pressure & $x, y, z$ & coordinates \\
$Q$ & local heat flux & $\delta$ & liquid film thickness \\
$Q_{C L}$ & heat flux in the contact line region & $\delta^{\prime}$ & derivative of $\delta$ with respect to $x$ \\
$Q_{i n t}$ & integrated heat flux & $\Delta h_{v}$ & enthalpy of evaporation \\
$Q_{M L}$ & heat flux in the microlayer region & $\Delta T$ & wall superheat \\
$Q_{w}$ & local heat flux across the wall & $\Delta T_{m e a n}$ & mean wall superheat within a set \\
$\tilde{Q}_{w}$ & median of $Q_{w}$ in time & $\theta$ & apparent contact angle \\
$R$ & thermal resistance of the liquid interface & $\mu$ & liquid viscosity \\
$t$ & time & $\rho_{s}$ & liquid density \\
$T$ & temperature & $\sigma$ & solid density \\
& & & liquid surface tension \\
\hline
\end{tabular}

The evaporation of these so-called microlayers leads to extremely large heat fluxes in this region (Kim, 2009). However, microlayers are not always observed and their formation process is therefore the subject of ongoing research. Furthermore, nucleate boiling is a highly transient process and the dewetting velocity during bubble growth depends on wall superheat, amongst other parameters. To the best knowledge of the authors a systematic and comprehensive study of these influencing parameters on microlayer formation during bubble growth is still lacking and very challenging due to the high demands on temporal and spatial resolution.

Fischer et al. (2015) showed that liquid films forming behind an evaporating receding meniscus result in a similar heat flux profile to the one of microlayers underneath growing vapor bubbles. Since the configuration studied by Fischer et al. (2015) is very similar to dip-coating, we will also refer to dip-coated evaporating films as microlayers in the following discussions. The dewetting situation studied in the work presented here is schematically illustrated in Fig. 1. In Fig. 1(a) a situation is sketched where the dewetting velocity $u$ is below $u_{c r i t}$. In this situation a macroscopic

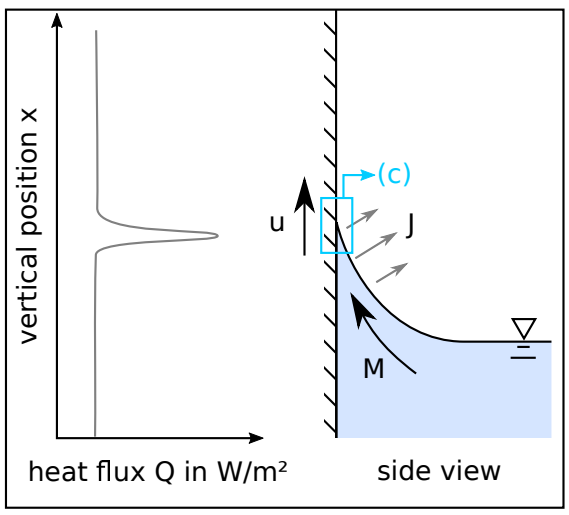

(a)

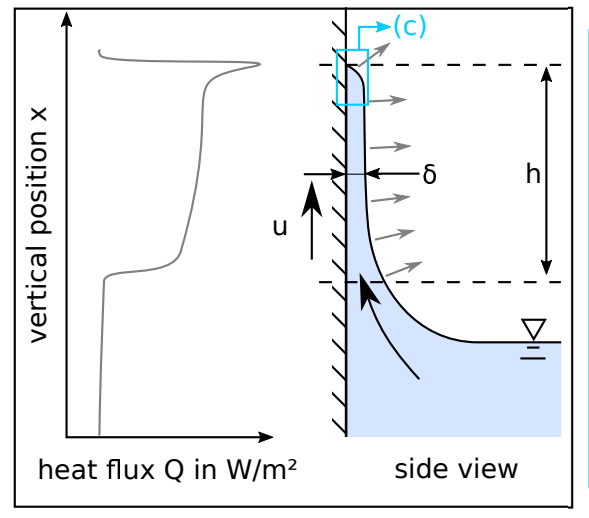

(b)

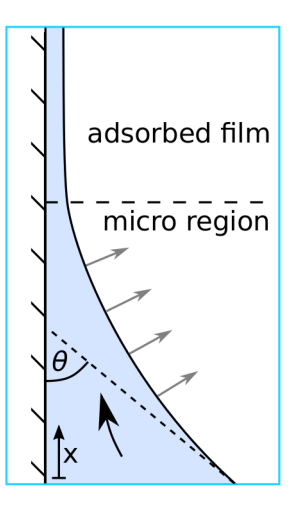

(c)

FIG. 1: Schematic heat flux profiles during the dewetting of a superheated wall: (a) contact line evaporation regime $\left(u<u_{\text {crit }}\right.$ ), (b) microlayer evaporation regime $\left(u>u_{\text {crit }}\right)$, (c) contact line region or micro region in (a) and (b) 
dynamic contact angle $\theta$ can be observed, and a sharp peak in heat flux occurs close to the three-phase contact line, where solid, liquid, and vapor phase meet. This tiny contact line region, also referred to as micro region, is shown enlarged in Fig. 1(c). The peak in local heat flux is explained by the local transition from the liquid pool to a thin adsorbed film, which leads to a strong local evaporation rate (Stephan and Hammer, 1994; Stephan and Busse, 1992). The situation with $u<u_{\text {crit }}$ shown in Fig. 1(a) is referred to as the contact line evaporation regime. The heat flux in the micro region increases with the local wall temperature and leads to a local cooling effect (Sodtke et al., 2008). Furthermore, the heat flux increases with the dewetting velocity, which is explained by a reduction in the contact angle, which in turn reduces the mean film thickness in the micro region. Note though, that an increased evaporation rate in the micro region generally causes a rise in contact angle (Anderson and Davis, 1995), therefore both effects act against each other. We point to Ajaev and Kabov (2017) for a more detailed analysis on the subject. In Fig. 1(b) a situation is sketched where the dewetting velocity $u$ exceeds $u_{\text {crit }}$. In this situation a liquid microlayer is formed on the substrate surface. The high heat flux area spreads all along this microlayer of length $h$, with a less pronounced peak that still occurs close to the contact line. This situation in Fig. 1(b) is referred to as the microlayer evaporation regime.

The aim of this paper is to investigate the influence of the dewetting velocity and the wall superheat on the heat flux profile at the interface wall-fluid. To achieve this, we measure the wall temperature distribution of the solidliquid and the solid-vapor interfaces using high-speed infrared thermography. The temperature data are then further used as a time- and space-resolved boundary condition to compute the transient heat flux profiles at the interfaces. These profiles are then used to analyze and discuss differences in local heat flux between contact line evaporation and microlayer evaporation.

\section{METHODS OF INVESTIGATION}

\subsection{Experimental Method}

The central element of the experimental setup is depicted in Fig. 2. It consists of an infrared transparent substrate (calcium fluoride, $\mathrm{CaF}_{2}$ ), which is covered by a thin double layer of chromium nitride $(\mathrm{CrN})$ and chromium $(\mathrm{Cr})$, each $500 \mathrm{~nm}$ in thickness. The $\mathrm{CaF}_{2}$ substrate is circular with a diameter of $40 \mathrm{~mm}$ and a thickness of $5 \mathrm{~mm}$, while the coating takes the shape of a $20 \mathrm{~mm}$ wide vertical strip across the center of the substrate. While the high emissivity of the $\mathrm{CrN}$ ensures a good infrared signal, the $\mathrm{Cr}$ layer is used for joule heating and provides resistance against mechanical stress. The substrate is mounted into a housing, which can be moved up or down with a set velocity $u$. One side of the housing and substrate is filled with the test fluid FC-72 (perfluorohexane, $\mathrm{C}_{6} \mathrm{~F}_{14}$ ) under saturated conditions, thus with a pool of liquid FC-72 and its vapor above. The FC-72 perfectly wets the chromium surface (static apparent contact angle below $10^{\circ}$ ), forming a liquid meniscus on the $\mathrm{Cr}$ layer. A seal between sample and housing prevents liquid from entering the other side of the housing, which therefore remains dry. The temperature field near the contact line is observed from the dry side of the substrate using a high-speed infrared camera at a frame

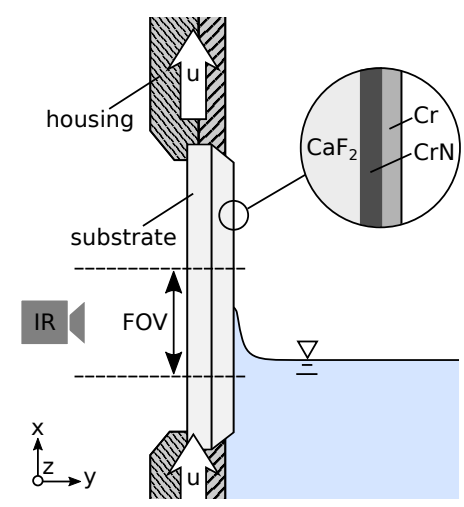

FIG. 2: Sketch of the central element of the experimental setup

Volume 7, Issue 3, 2019 
rate of $1004 \mathrm{~Hz}$, a resolution of $512 \times 640$ pixels and a pixel size of $25 \mu \mathrm{m}$. The total field of view of the infrared camera (FOV) measures $12.8 \mathrm{~mm}$ by $16 \mathrm{~mm}$. During the experiment, the substrate is heated by applying a current to the $\mathrm{Cr}$ layer and then moved upwards out of the liquid pool through the FOV of the infrared camera. While the liquid pool, the infrared camera, and the FOV remain in fixed positions, a receding contact line is formed on the moving substrate. During this process, only the small part of the moving substrate currently located in the FOV (i.e., the part where the dewetting process is currently taking place) is observed. All experiments presented were performed at saturated conditions at a liquid and vapor temperature of $25^{\circ} \mathrm{C}$, which corresponds to a system pressure of 290 mbar. Further details on the experimental setup including the thermalization, etc. and the calibration procedure of the infrared camera are reported in Schweikert et al. (2019).

\subsection{Numerical Method}

In order to derive the heat flux at the interface between solid wall and liquid/vapor phase, the temperature distribution inside the solid heater substrate is computed using the measured temperature data at the solid-fluid interface as a boundary condition. The computational domain is illustrated in Fig. 3, in which the FOV of the infrared camera and all domain boundaries are also shown. Since the thicknesses of the $\mathrm{Cr}$ and $\mathrm{CrN}$ layers are extremely small, they can be neglected in the calculations. Thus the solid substrate is modeled as pure $\mathrm{CaF}_{2}$ and the measured temperature field is placed directly on the $\mathrm{CaF}_{2}$ surface at boundary $\mathrm{B} 3$. Since the measured temperature field is highly homogeneous in the transverse direction ( $z$ direction), the problem can be reduced to two dimensions. This is done by averaging the temperature measurements in $z$ direction across a $7.5 \mathrm{~mm}$ wide area located in the center of the sample. This reduces temporal noise inherently present in the infrared data and the necessity for strong filtering operations is avoided. Since the area over which it is averaged is located in the center of the sample and is much smaller than both the sample or the coated area, boundary effects can be assumed to be negligible. Calculations are done using COMSOL Multiphysics 5.4 (Build: 295). Since the measured temperature profile is only available in the FOV at boundary B3, calculations are not carried out for the whole solid substrate at once. Only a portion around the FOV is represented by the discretization as shown in Fig. 3. The movement of the solid substrate is accounted for by the convective termin the energy equation, Eq. (1), which is solved for every cell and time step. Vectors are represented in bold font.

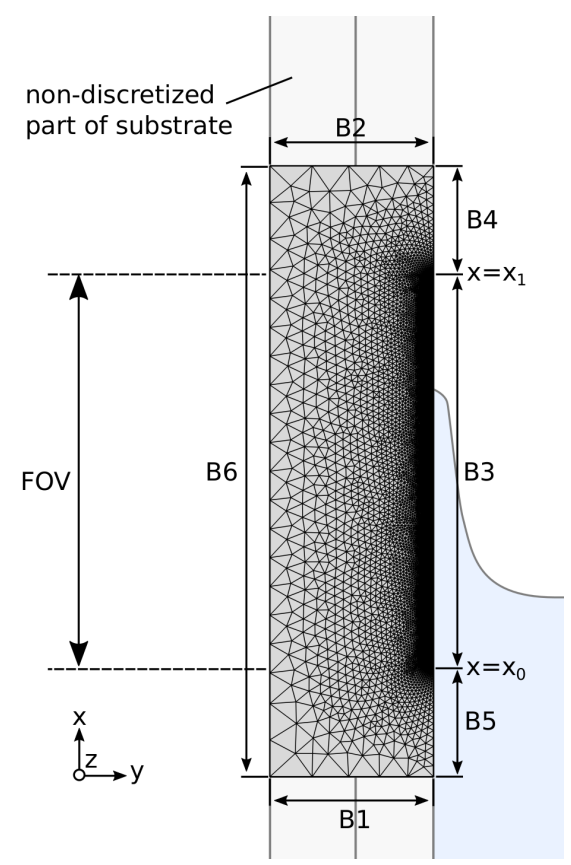

FIG. 3: Computational domain including grid and boundaries 


$$
\rho_{s} c_{s} \frac{\partial T}{\partial t}+\rho_{s} c \mathbf{u} \nabla T+k_{s} \nabla^{2} T=0
$$

In Eq. (1), $\mathbf{u}_{x}$ is set to the vertical dewetting velocity $u$. This way, the necessity for a moving boundary condition is avoided. The coefficients $c_{s}, \rho_{s}$, and $k_{s}$ describe the substrate's specific heat capacity, density, and thermal conductivity, respectively. They are set to those of solid calcium fluoride at $25^{\circ} \mathrm{C}$ and assumed constant $\left(c_{s}=854\right.$

$\mathrm{J} \mathrm{kg}^{-1} \mathrm{~K}^{-1}, \rho_{s}=3.18 \mathrm{~g} \mathrm{~cm}^{-3}, k_{s}=9.71 \mathrm{~W} \mathrm{~m}^{-1} \mathrm{~K}^{-1}$ ). Information about the size of the computational domain and grid, as well as the boundary conditions are summarized in Table 1. The measured temperature data are used as a time-dependent boundary condition on boundary B3. As the pixel size of the infrared camera is more than twice as large as the size of the cells at B3, the boundary between two pixels can lie within one cell. For these cells, the local value for the temperature boundary condition is calculated by linear interpolation in between the pixel centers. The calculation area is extended above and below B3 to gain an estimation of the temperature field outside of the FOV. Temperatures on boundaries B4 and B5 are set to the measured temperature value at $x_{1}$ and $x_{0}$, respectively. This can be seen as valid, as long as the vertical temperature gradient at $x=x_{0}$ and $x=x_{1}$ can be assumed zero. Since the temperature field above and below the FOV is calculated, this assumption can be checked after the calculations. Experiments, in which the thermal gradient cannot be assumed zero, are then excluded from further analysis. This is the case, for example, if the microlayer grows too long and the contact line reaches near $x=x_{1}$. Boundaries B1 and B2 experience a mass in- and outflux, respectively, due to the convective term representing the movement of the substrate. Their thermal boundary conditions are set to zero gradient for the reasons explained above. Boundary B6 is assumed to be adiabatic, because heat losses to the low-pressure atmosphere at this boundary can be neglected. The initial substrate temperature is set to the measured wall temperature below the contact line $\left[T_{w}\right.$ in Fig. 4(b)]. The whole computational domain is meshed using a triangular grid with a minimum element size at boundary B3, gradually increasing in size towards regions further away. A mesh refinement study was conducted to ensure grid independence of the solution. The numerical calculation results in a time-dependent temperature field within the solid substrate, from which the heat flux between the $\mathrm{CaF}_{2}$ and the $\mathrm{CrN} / \mathrm{Cr}$ coating, $Q_{\mathrm{B} 3}(x)$, is calculated. Within the coating, $Q_{e}$ describes the homogeneous heat source due to continuous joule heating during the experiment. Since the coating is negligibly thin, the heat flux across the solid-liquid/solid-vapor interface is calculated as $Q_{w}(x)=Q_{\mathrm{B} 3}(x)+Q_{e}$.

\subsection{Data Analysis}

Figure 4 shows the results obtained with the described process from an arbitrary measurement for one time step. All figures share the same vertical axis and Figs. 4(b) and 4(c) also share the same color scale. A schematic side view of the dip-coating process is shown in Fig. 4(a) for explanatory purposes, depicting the microlayer above the bulk liquid on the moving wall. The instantaneous temperature field gained through infrared thermography is shown in Fig. 4(b), in which three extensive regions can be identified: the bulk liquid region, the microlayer region, and the dry wall region. The wall temperature is highest in the bulk region and used to define the wall superheat $\Delta T=T_{w}-T_{\text {sat }}$. Due to evaporation in the microlayer region, temperatures decrease in the microlayer region and reach a minimum in the contact line region. Above the contact line, a dry wall remains. The coordinate $x$ is chosen to describe the distance

TABLE 1: Geometry and boundary conditions of the calculation area

\begin{tabular}{|ccccc|}
\hline Boundary name & Size in mm & $\begin{array}{c}\text { Number of } \\
\text { elements }\end{array}$ & $\begin{array}{c}\text { Thermal boundary } \\
\text { condition }\end{array}$ & $\begin{array}{c}\text { Convective boundary } \\
\text { condition }\end{array}$ \\
\hline B1, B2 & 5 & 5 & $\nabla T=0$ & $u_{x}=u ; u_{y}=0$ \\
B3 & 11.55 & 1024 & $T=f(x, t)_{\text {measured }}$ & $u_{x}=u ; u_{y}=0$ \\
B4 & 3.5 & 35 & $T=f\left(x_{1}, t\right)_{\text {measured }}$ & $u_{x}=u ; u_{y}=0$ \\
B5 & 3.5 & 35 & $T=f\left(x_{0}, t\right)_{\text {measured }}$ & $u_{x}=u ; u_{y}=0$ \\
B6 & 18.66 & 20 & adiabatic & $u_{x}=u ; u_{y}=0$ \\
\hline
\end{tabular}

Volume 7, Issue 3, 2019 


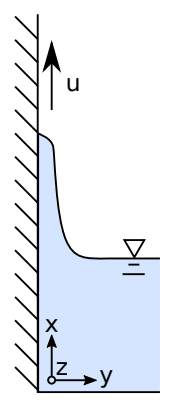

(a)

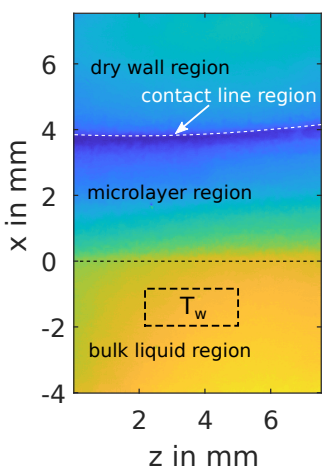

(b)

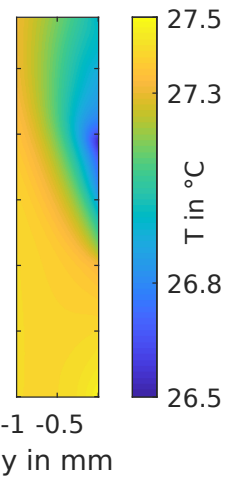

(c)

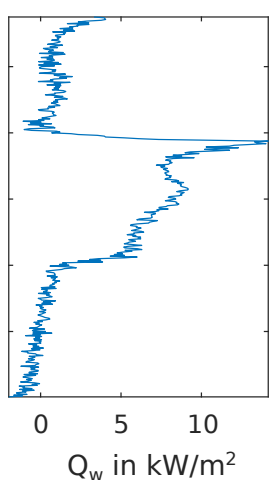

(d)

FIG. 4: Exemplary overview of a measurement and results of the numerical calculation at $t=0.42 \mathrm{~s}$ after starting the withdrawal process $\left(u=28.16 \mathrm{~mm} \mathrm{~s}^{-1}, \Delta T=2.55 \mathrm{~K}, Q_{e}=1.37 \mathrm{~kW} \mathrm{~m}^{-2}\right)$. (a) schematic side view, (b) measured temperature field at the wall-liquid interface, (c) calculated temperature field inside the wall, (d) evaluated local heat flux across boundary B3.

above the bulk liquid region, where the origin of $x$ is found by locating the contact line in a static reference image taken before the experiment $(u=0) . y$ describes the coordinate perpendicular to the wall/fluid interface, with negative values of $y$ describing locations inside the solid substrate. Within every time step the measured temperature field is averaged along the $z$ direction and used as a boundary condition (at boundary B3) for the time-dependent calculation of the temperature field inside the wall, which is shown in Fig. 4(c) for the same time step. The cooling effect of the microlayer extends roughly $1 \mathrm{~mm}$ into the wall and the high decrease of the local temperature in the contact line region is clearly visible. The corresponding heat flux across the wall is shown in Fig. 4(d), where positive values indicate heat flux from the wall towards the fluid. Negative values can be observed near $x<0$, which are a result of assuming uniform joule heating across the sample, even though it slightly increases close to the sample's edge. As this area is not used for the evaluation of any quantities in our analysis, we assume this effect to be negligible. To compare the time-dependent results of different measurements with one another, a single heat flux profile $\tilde{Q}_{w}(x)$ is calculated for each measurement using the temporal median of the time dependent heat flux profiles. Only timesteps are taken into account, in which the dewetting situation reached quasi-steady state, i.e., a finite microlayer length has been reached. The growth of the microlayer at the beginning of each measurement sequence is omitted. We point to our analysis of the microlayer length and its temporal fluctuation for further information (Schweikert et al., 2019). The measurement uncertainty of the dewetting velocity can be estimated to $0.15 \mathrm{~mm} \mathrm{~s}^{-1}$ accuracy, while the measured fluid, gas, and wall temperature underlie a base uncertainty of $0.1 \mathrm{~K}$. Experiments were carried out for a range of dewetting velocities, while the wall superheat was kept constant. This was repeated for a total of six levels of wall superheat. However, the wall superheat cannot be perfectly reproduced in each experiment. The standard deviation of wall superheat between single experiments within a set is therefore given in Table 2 . Therein $\Delta T_{\text {mean }}$ describes the mean wall superheat of a single set, which is used in the legend of later figures. The exact value of $u_{\text {crit }}$ is generally unknown. Therefore, throughout the following discussion, we base our assumption of whether $u_{\text {crit }}$ is exceeded on the observation of a microlayer within the infrared images.

TABLE 2: Mean wall superheat $\Delta T_{\text {mean }}$ and corresponding standard deviation within a set of experiments grouped by their similarity in $\Delta T$

\begin{tabular}{|ccccccc|}
\hline Set number & $\mathbf{\# 1}$ & $\mathbf{\# 2}$ & $\mathbf{\# 3}$ & $\mathbf{\# 4}$ & $\mathbf{\# 5}$ & $\mathbf{\# 6}$ \\
\hline$\Delta T_{\text {mean }}$ in $\mathrm{K}$ & 1.44 & 1.54 & 2.01 & 2.55 & 3.47 & 4.08 \\
Deviation in K & 0.04 & 0.04 & 0.17 & 0.05 & 0.07 & 0.13 \\
\hline
\end{tabular}




\section{RESULTS AND DISCUSSION}

\subsection{Heat Flux Profile Shapes}

Figure 5 shows the median heat flux profiles $\tilde{Q}_{w}$ at two different values for the wall superheat. The coordinate $x$, corresponding to the distance above the bulk liquid region, is shown on the abscissa. The influence of the dewetting velocity $u$ on the microlayer formation is clearly visible: For the lowest dewetting velocities shown, only a small peak in heat flux can be observed close to the bulk region (e.g., $\Delta T_{\text {mean }}=2.55 \mathrm{~K}, u=13.06 \mathrm{~mm} \mathrm{~s}^{-1}$ ). As explained in Fig. 1(a), this peak is characteristic for the contact line evaporation regime. Indeed, no microlayer formation was observed during these measurements. For $u<u_{\text {crit }}$, the position of this peak slightly shifts further away from the bulk region with increasing $u$, as can be seen when comparing the two lowest dewetting velocities $\left(u=7.5 \mathrm{~mm} \mathrm{~s}^{-1}\right.$ and $u=18.62 \mathrm{~mm} \mathrm{~s}^{-1}$ for $\Delta T_{\text {mean }}=3.47 \mathrm{~K}$ ). Based on the analysis of Herbert et al. (2013), we assume that this shift is due to a decrease in dynamic contact angle with increasing dewetting velocity. The magnitude of this peak is discussed in Section 3.3. As expected, the shape of the heat flux profile changes once a microlayer forms $\left(u>u_{\text {crit }}\right)$. Again, the heat flux quickly rises above the bulk liquid region. The peak heat flux is still located near the contact line, but the value is roughly twice as high as in the contact line evaporation regime. In the elongated microlayer region the heat flux profile has a characteristic shape with increasing heat flux values towards the contact line. As the dewetting velocity $u$ increases, the microlayer grows in length and the heat flux profile in this region takes the shape of a plateau, as it was already described qualitatively in Fig. 1(b). With increasing microlayer length the heat flux peak near the contact line slightly decreases and becomes broader. This is largely due to temporal fluctuations in $h$ and the position of the contact line is therefore not constant during the measurement. When calculating the median heat flux profiles, the peak therefore appears lower, but with its base spread over a larger area.
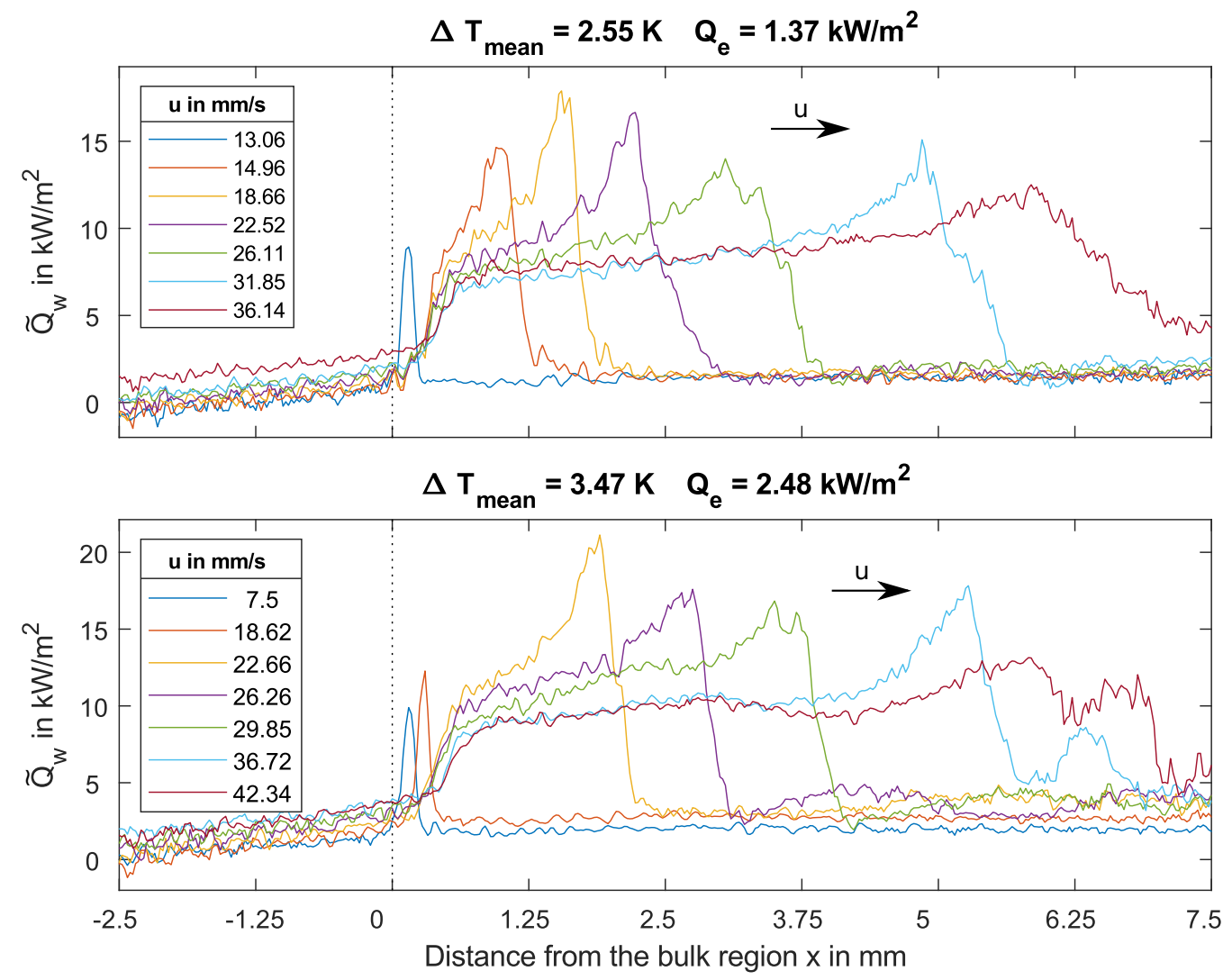

FIG. 5: Median heat flux profiles for different dewetting velocities

Volume 7, Issue 3, 2019 


\subsection{Integrated Heat Flux}

To directly compare the overall heat transferred through the microlayer region for different dewetting velocities and/or different wall superheats, we reduce each median heat flux profile $\tilde{Q}_{w}$ (shown in Fig. 5) by integrating it from the end of the bulk liquid region $(x=0)$ to the start of the dry wall region $\left(x=x_{2}\right)$, gaining the integrated heat flux $Q_{\text {int }}$. The results are shown in Fig. 6, where $Q_{\text {int }}$ is plotted over the dewetting velocity $u$ for different wall superheats $\Delta T_{m e a n}$. All curves at constant $\Delta T_{\text {mean }}$ follow the same trend: First, at low dewetting velocity, $Q_{\text {int }}$ remains low, until a threshold value in $u$ is exceeded. Here $Q_{i n t}$ experiences a sharp rise in magnitude, after which $Q_{\text {int }}$ steadily grows with increasing dewetting velocity. The threshold value marks $u_{c r i t}$ and therefore the transition between the contact line evaporation regime and the microlayer evaporation regime. The dependency of $u_{c r i t}$ on $\Delta T$ is clearly visible in the regime change shifting towards higher velocities as $\Delta T_{\text {mean }}$ is increased. Since the experiments presented here were not conducted specifically for the determination of $u_{c r i t}$, the value of $u_{c r i t}$ for any given $\Delta T_{\text {mean }}$ is assumed equal to the dewetting velocity at the last data point, where no microlayer was observed. Using these data points, the dependency of $u_{\text {crit }}$ on $\Delta T_{\text {mean }}$ is approximately linear and can be estimated with $u_{\text {crit }} \approx 8.08 \Delta T-7.42$. Since the velocity dependency of $Q_{i n t}$ is stronger in the microlayer evaporation regime than in the contact line evaporation regime, the jump in heat flux becomes larger at higher values of $\Delta T_{\text {mean }}$. All curves line up very nicely, both in the contact line evaporation regime and in the microlayer evaporation regime. The result seems counterintuitive, as the microlayer length $h$ is very different for many data points in the microlayer evaporation regime in Fig. 6 that yield nearly the same $Q_{\text {int }}$. In Fig. $7 Q_{\text {int }}$ is therefore plotted over the microlayer length $h$. As $h$ decreases with growing $\Delta T$, it is evident that the same $Q_{i n t}$ can be achieved at different $\Delta T$. From this figure, one might expect that an increase in $\Delta T$ always leads to an increase in $Q_{i n t}$. However, in the microlayer evaporation regime $Q_{i n t}$ is limited by the amount of liquid being entrained into the microlayer by the motion of the wall. Since the thickness of the liquid film in the microlayer region scales $\delta \sim u^{2 / 3}$ (Schweikert et al., 2019), the integral mass flow $M$ scales $M \approx u \delta \sim u^{5 / 3}$. Since $Q_{\text {int }}$ represents the heat flux caused by the whole microlayer and therefore all of $M$, the scalings must also be the same. Indeed, fitting a power law to $Q_{i n t}$ in the microlayer evaporation regime yields a very similar exponent of 1.65. In the microlayer evaporation regime $Q_{i n t}$ is therefore only a function of $u$ and independent of the wall superheat $\Delta T$, provided no regime change is caused by the change of $\Delta T$. If a change into the contact line evaporation regime does occur, however, an increase in $\Delta T$ always has a negative effect on the heat transfer. For instance, increasing $\Delta T_{\text {mean }}$ from 3.47 to $4.08 \mathrm{~K}$ at a velocity of $22.66 \mathrm{~mm} \mathrm{~s}^{-1}$ causes a change from the microlayer evaporation regime to the contact line evaporation regime, which results in a reduction of $Q_{\text {int }}$ by roughly $85 \%$.

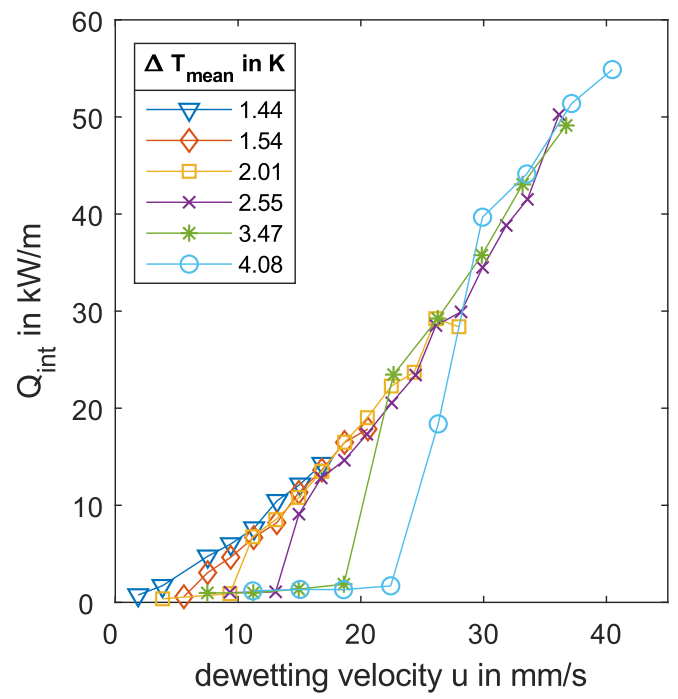

FIG. 6: Integrated heat flux $Q_{\text {int }}$ over the dewetting velocity $u$ at different wall superheat $\Delta T_{m e a n}$ 


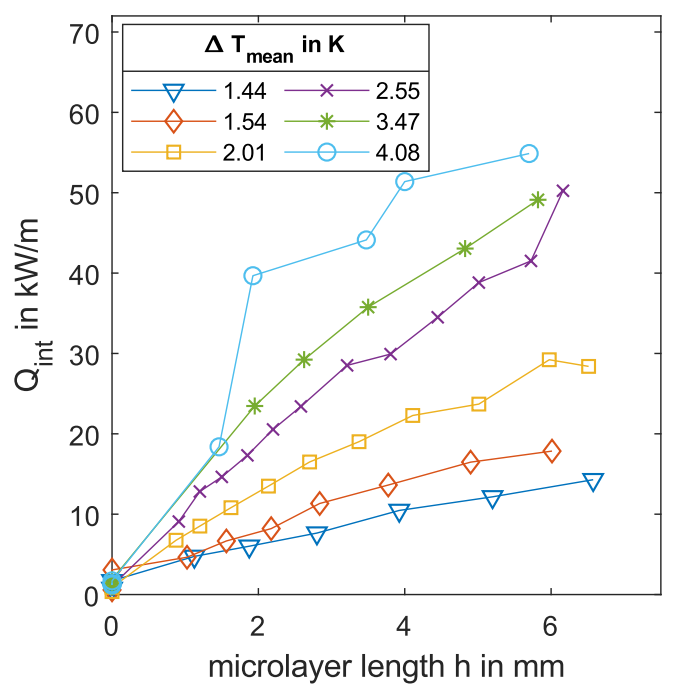

FIG. 7: Integrated heat flux $Q_{i n t}$ over the microlayer length $h$ at different wall superheat $\Delta T_{\text {mean }}$

\subsection{Heat Flux near the Contact Line}

Figure 8 shows the median heat flux in the contact line region, $Q_{C L}$, over the velocity difference $u-u_{c r i t}$, where $u$ is the dewetting velocity and $u_{\text {crit }}$ the critical velocity for the regime transition. Thus, negative values of $u-u_{\text {crit }}$ correspond to the contact line evaporation regime, while positive values correspond to the microlayer evaporation regime. Since the contact line region described in Section 1 [Fig. 1(c)] is much smaller than the pixel size of the infrared camera used in the experiments, $Q_{C L}$ is evaluated based on the maximum of the time-dependent heat flux profiles, rather than the median heat flux. To avoid the motion of the contact line to affect the analysis, $Q_{C L}$ is first evaluated for every time step, from which the median is then calculated. $Q_{C L}$ presented in Fig. 8 does therefore not exactly equal the peak value of the heat flux profiles shown in Fig. 5. As can be seen in Fig. 8, $Q_{C L}$ sharply rises

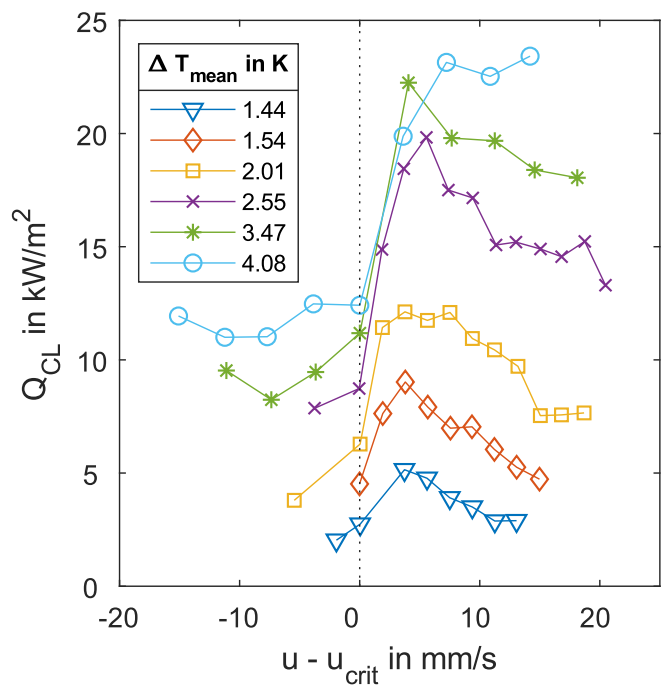

FIG. 8: Median heat flux at the contact line $Q_{C L}$ over the dewetting velocity difference $u-u_{\text {crit }}$ 
when changing into the microlayer evaporation regime at $u=u_{\text {crit }}$ and a peak can be observed shortly after. Note that large values of $u-u_{\text {crit }}$ also correspond to long microlayer lengths, because the microlayer length scales $h \sim u^{5 / 3}$. We can therefore conclude that the heat flux in the contact line region, $Q_{C L}$, is maximum when the microlayer is particularly short. The question arises, why such a sharp rise in heat flux near the contact line is observed during the regime change. To estimate general dependencies, we follow Batzdorf (2015) for an expression for the heat flux in the contact line region, Eq. (2):

$$
Q_{C L}=\left[T_{C L}-T_{\text {sat }}\left(1+\frac{P}{\Delta h_{v} \rho}\right)\right] /\left(\frac{\delta}{k}+\frac{R}{\sqrt{1+\delta^{\prime 2}}}\right)
$$

Therein $k$ describes the thermal conductivity of the liquid film, $\delta$ its thickness, $R$ the thermal resistance at the liquid-vapor interface, and $\Delta h_{v}$ the specific enthalpy of vaporization. $P$ describes the local capillary pressure, $T_{C L}$ the local substrate temperature in the contact line region, and $\delta^{\prime}$ the derivative of $\delta$ with respect to $x$. For further discussion, we assume all quantities except for $T_{C L}, P$, and $\delta$ to be constant within the presented experiments. One might expect the sharp increase of $Q_{C L}$ at $u_{c r i t}$ to be caused by an increase in $T_{C L}$. Figure 9 therefore shows the median wall superheat in the contact line region $\Delta T_{C L}=T_{C L}-T_{\text {sat }}$ scaled by $\Delta T=T_{w}-T_{\text {sat }}$. The ratio $\Delta T_{C L} / \Delta T$ changes significantly at the regime boundary, therefore the effect seen in Fig. 8 cannot be temperature driven. The increased cooling of the wall is therefore either caused by a strong decrease in the local liquid film thickness in the contact line region, or a decrease in capillary pressure. As mentioned, Herbert et al. (2013) showed that the contact angle on a superheated wall decreases with increasing dewetting velocity. This results in a lower film thickness in the contact line region and in turn in an increased heat flux near the contact line. Extrapolating this trend to $u_{c r i t}$, where the contact angle can be expected to reach a minimum, a further rise in heat flux seems plausible. However, in the results of Herbert et al. (2013) the increase in heat flux occurs gradually, which is not seen in the experiments. The capillary pressure in the contact line region increases as the curvature of the liquid-vapor interface decreases. For small contact angles it is therefore expected that the numerator of Eq. (2) reduces. Since an increase in $Q_{C L}$ is seen in the experiments, however, $\delta$ must significantly reduce at $u_{\text {crit }}$ to counteract the reductive effects of $T_{C L}$ and $P$. A plausible explanation can be drawn from the results of Qu et al. (2002), who measured a very thin $(\approx 0.1 \mu \mathrm{m})$ "foot" at end of their evaporating liquid films, similar to a precursor film. Since our microlayers and the liquid films of Qu et al. (2002) share the same trends for $h$ and $\delta$ and have comparable fluid properties, it is likely

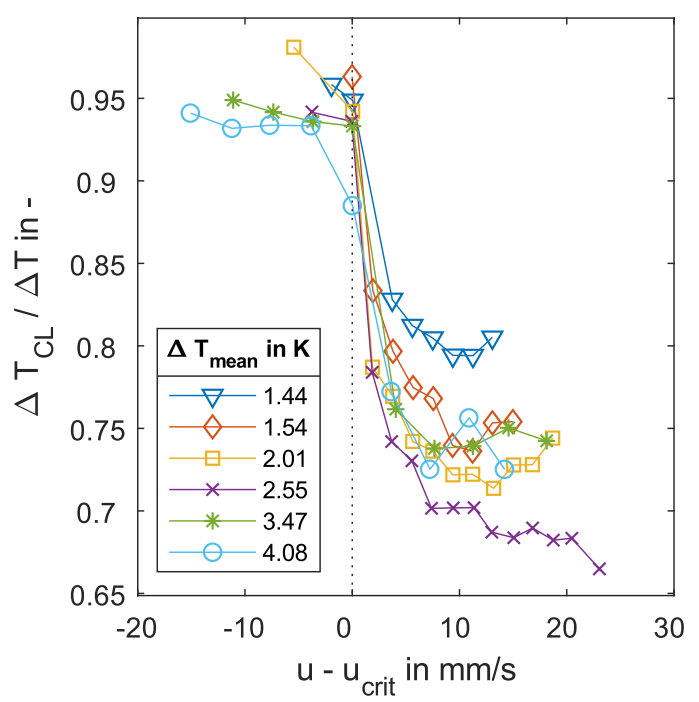

FIG. 9: Ratio between the local wall superheat at the contact line $\Delta T_{C L}$ and the wall superheat $\Delta T_{m e a n}$ over the dewetting velocity difference $u-u_{\text {crit }}$ 
that a similar precursor film exists at the end of the microlayers described here. A region of large curvature connects this foot with the rest of the film, which would reduce the local capillary pressure in the contact line region. To be responsible for the sudden increase in heat flux, the foot has to start forming when $u_{\text {crit }}$ is exceeded, enhancing heat transfer from then on near the contact line. As $Q_{C L}$ does not further rise with $u>u_{c r i t}$, the dependency of this film geometry on $u$ must be very weak.

Last we discuss the drop in $Q_{C L}$ with increasing microlayer length. One would expect $Q_{C L}$ to further rise with $u$ based on the previous discussion about a decreasing contact angle with rising $u$. We therefore assume that a minimum in contact angle is reached at $u_{c r i t}$, preventing a further rise in $Q_{C L}$ with $u$. Consequently, the contact angle at the end of the microlayer is either the same or higher than the contact angle reached at $u=u_{\text {crit }}$. The decrease in $Q_{C L}$ at further increase of $u$ can be explained when evaluating the integral value around $Q_{C L}$. This integral quantity does not show a strong decline after $u_{c r i t}$ is surpassed. The observed drop in $Q_{C L}$ does therefore not necessarily indicate that heat flux in the contact line region strongly declines, but rather that it is spread over a larger area as $u$ increases. Furthermore, at higher $u-u_{\text {crit }}$ a longer microlayer exists above the bulk liquid region, cooling the wall before it reaches the contact line region. This is noticeable in Fig. 9, where $\Delta T_{C L}$ continues to drop in the microlayer evaporation regime with increasing $u$, which slightly reduces the driving force for evaporation in the contact line region. The decline in $Q_{C L}$ at velocities above $u_{c r i t}$ is most likely a combination of the two described effects.

\subsection{Heat Flux in the Microlayer Region}

Figure 10 shows the median heat flux in the microlayer region $Q_{M L}$ over the microlayer length $h$. A slight decrease can be seen with increasing $h$, which we account to two effects. First, both $\delta$ and $h$ increase with the dewetting velocity. At constant $\Delta T$, longer microlayers are therefore thicker, which increases the heat conduction resistance through the liquid film and results in a lower evaporation rate. Second, short microlayers experience a larger gradient in heat flux, from a minimum near the bulk liquid region to a maximum near the contact line region. $Q_{C L}$ decreases with increasing $h$ as discussed previously. As the microlayer gets longer, the heat flux profile of the microlayer becomes flatter and the heat flux in the contact line region decreases in relation to the rest of the microlayer. To compare both regions of the microlayer, Fig. 11 shows the ratio $Q_{C L} / Q_{M L}$ over the microlayer length $h$. The ratio is highest for short microlayers and falls as $h$ increases. Contact line evaporation is therefore most important for short microlayers, where the local evaporation rate is roughly twice as high as in the rest of the microlayer.

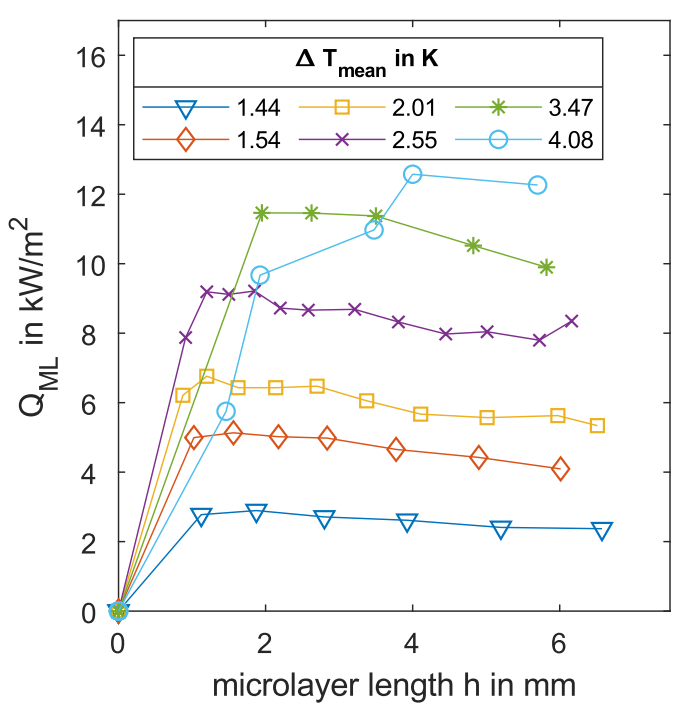

FIG. 10: Median heat flux in the microlayer $Q_{M L}$ over the microlayer length $h$ at different wall superheat $\Delta T_{m e a n}$

Volume 7, Issue 3, 2019 


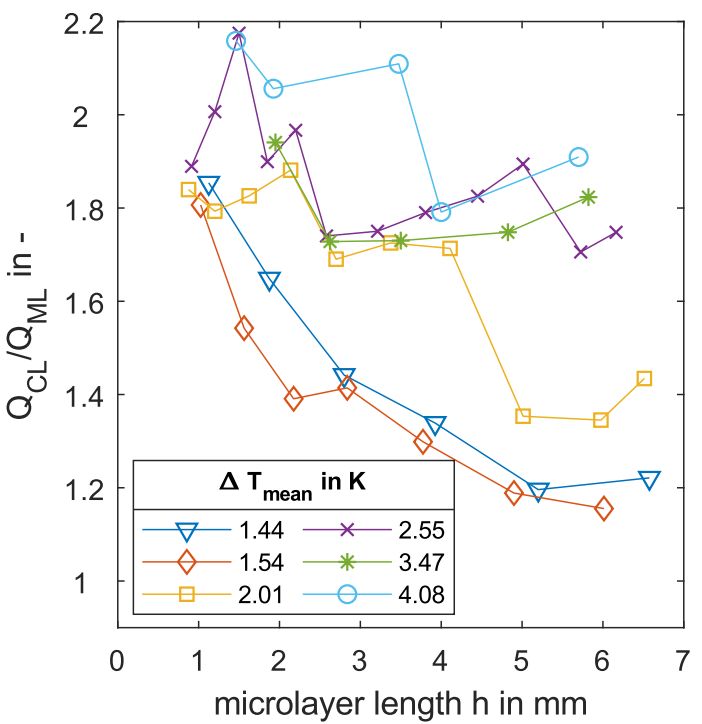

FIG. 11: Comparison of the median heat flux in the microlayer $Q_{M L}$ to the median heat flux at the contact line $Q_{C L}$ over the microlayer length $h$ at different wall superheat $\Delta T_{\text {mean }}$

\section{CONCLUSION}

In this paper we have analyzed the heat flux profiles occurring during the dip-coating process of a superheated substrate. The influence of wall superheat and dewetting velocity on the integrated heat flux, as well as the contribution of the microlayer region and contact line region were investigated. We can summarize our conclusions as follows:

1. Two evaporation regimes, contact line evaporation and microlayer evaporation, can be clearly distinguished. They are separated by a temperature-dependent critical dewetting velocity. The change between both regimes is sudden and alters the overall heat flux between wall and liquid considerably.

2. In the microlayer evaporation regime, the overall heat flux scales $Q_{i n t} \sim u^{5 / 3}$ and is almost independent of the wall superheat.

3. The local heat flux in the contact line region increases sharply once the dewetting velocity exceeds the critical dewetting velocity. In the microlayer evaporation regime, the local heat flux in the contact line region is highest for short microlayers and slowly decreases as the microlayer length grows.

Since the increased heat transfer in the contact line region could not be fully explained, the contact line region should be studied in more detail near the regime change. Detailed measurements of the microlayer's thickness profile are therefore planned for the future. In addition, numerical simulations of the regime change could help to generate valuable insight into the microscopic flow profile near the contact line.

\section{ACKNOWLEDGMENTS}

We kindly acknowledge the financial support by the German Research Foundation (DFG) within the Collaborative Research Center 1194 "Interaction of Transport and Wetting Processes," project A01. The measurement data presented here are available at the following URL: https://tudatalib.ulb.tu-darmstadt.de/handle/tudatalib/2129. 


\section{REFERENCES}

Ajaev, V.S. and Kabov, O.A., Heat and Mass Transfer near Contact Lines on Heated Surfaces, Int. J. Heat Mass Transf., vol. 108, pp. 918-932, 2017.

Anderson, D.M. and Davis, S.H., The Spreading of Volatile Liquid Droplets on Heated Surfaces, Phys. Fluids, vol. 7, no. 2, pp. 248-265, 1995.

Batzdorf, S., Heat Transfer and Evaporation during Single Drop Impingement onto a Superheated Wall, PhD, TU Darmstadt, 2015.

Berteloot, G., Pham, C.T., Daerr, A., Lequeux, F., and Limat, L., Evaporation-Induced Flow near a Contact Line: Consequences on Coating and Contact Angle, EPL (Europhys. Lett.), vol. 83, no. 1, p. 14003, 2008.

Fischer, S., Gambaryan-Roisman, T., and Stephan, P., On the Development of a Thin Evaporating Liquid Film at a Receding Liquid/Vapour-Interface, Int. J. Heat Mass Transf., vol. 88, pp. 346-356, 2015.

Herbert, S., Fischer, S., Gambaryan-Roisman, T., and Stephan, P., Local Heat Transfer and Phase Change Phenomena during Single Drop Impingement on a Hot Surface, Int. J. Heat Mass Transf., vol. 61, pp. 605-614, 2013.

Hocking, L.M., Meniscus Draw-Up and Draining, Euro. J. Appl. Math., vol. 12, no. 3, pp. 195-208, 2001.

Kim, H. and Buongiorno, J., Detection of Liquid-Vapor-Solid Triple Contact Line in Two-Phase Heat Transfer Phenomena Using High-Speed Infrared Thermometry, Int. J. Multiphase Flow, vol. 37, no. 2, pp. 166-172, 2011.

Kim, J., Review of Nucleate Pool Boiling Bubble Heat Transfer Mechanisms, Int. J. Multiphase Flow, vol. 35, no. 12, pp. 10671076, 2009.

Landau, L. and Levich, B., Dragging of a Liquid by a Moving Plate, Acta Physicochim, vol. 17, pp. 42-54, 1942.

Qu, D., Rame', E., and Garoff, S., Dip-Coated Films of Volatile Liquids, Phys. Fluids, vol. 14, no. 3, pp. 1154-1165, 2002.

Rio, E. and Boulogne, F., Withdrawing a Solid from a Bath: How Much Liquid is Coated?, Adv. Colloid Interface Sci., vol. 247, pp. 100-114, 2017.

Schweikert, K., Sielaff, A., and Stephan, P., On the Transition between Contact Line Evaporation and Microlayer Evaporation during the Dewetting of a Superheated Wall, Int. J. Therm. Sci., vol. 145, p. 106025, 2019.

Sodtke, C., Ajaev, V.S., and Stephan, P., Dynamics of Volatile Liquid Droplets on Heated Surfaces: Theory versus Experiment, $J$. Fluid Mech., vol. 610, pp. 343-362, 2008.

Stephan, P. and Hammer, J., A New Model for Nucleate Boiling Heat Transfer, Wärme- und Stoffübertragung, vol. 30, no. 2, pp. 119-125, 1994.

Stephan, P.C. and Busse, C.A., Analysis of the Heat Transfer Coefficient of Grooved Heat Pipe Evaporator Walls, Int. J. Heat Mass Transf., vol. 35, no. 2, pp. 383-391, 1992.

Utaka, Y., Hu, K., Chen, Z., and Morokuma, T., Measurement of Contribution of Microlayer Evaporation Applying the Microlayer Volume Change during Nucleate Pool Boiling for Water and Ethanol, Int. J. Heat Mass Transf., vol. 125, pp. 243-247, 2018.

Volume 7, Issue 3, 2019 